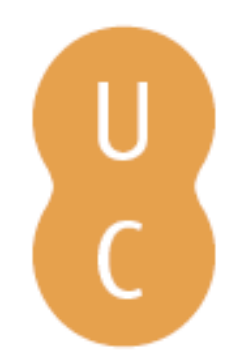

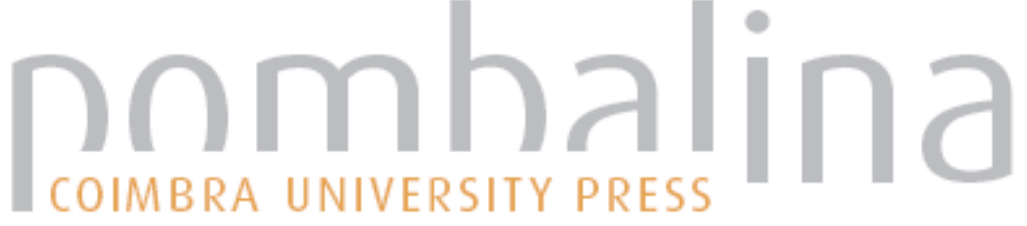

\section{Buscar o mar pela montanha}

\author{
Autor(es): $\quad$ Sartori, Antonio
}

Publicado por: Imprensa da Universidade de Coimbra

URL

persistente: $\quad$ URI:http://hdl.handle.net/10316.2/38349

DOI: $\quad$ DOI:http://dx.doi.org/10.14195/978-989-26-0438-1_9

Accessed : $\quad$ 26-Apr-2023 12:15:25

A navegação consulta e descarregamento dos títulos inseridos nas Bibliotecas Digitais UC Digitalis, UC Pombalina e UC Impactum, pressupõem a aceitação plena e sem reservas dos Termos e Condições de Uso destas Bibliotecas Digitais, disponíveis em https://digitalis.uc.pt/pt-pt/termos.

Conforme exposto nos referidos Termos e Condições de Uso, o descarregamento de títulos de acesso restrito requer uma licença válida de autorização devendo o utilizador aceder ao(s) documento(s) a partir de um endereço de IP da instituição detentora da supramencionada licença.

Ao utilizador é apenas permitido o descarregamento para uso pessoal, pelo que o emprego do(s) título(s) descarregado(s) para outro fim, designadamente comercial, carece de autorização do respetivo autor ou editor da obra.

Na medida em que todas as obras da UC Digitalis se encontram protegidas pelo Código do Direito de Autor e Direitos Conexos e demais legislação aplicável, toda a cópia, parcial ou total, deste documento, nos casos em que é legalmente admitida, deverá conter ou fazer-se acompanhar por este aviso.

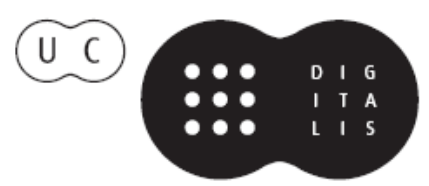


Francisco de Oliveira

Pascal Thiercy

Raquel Vilaça

Coordenação

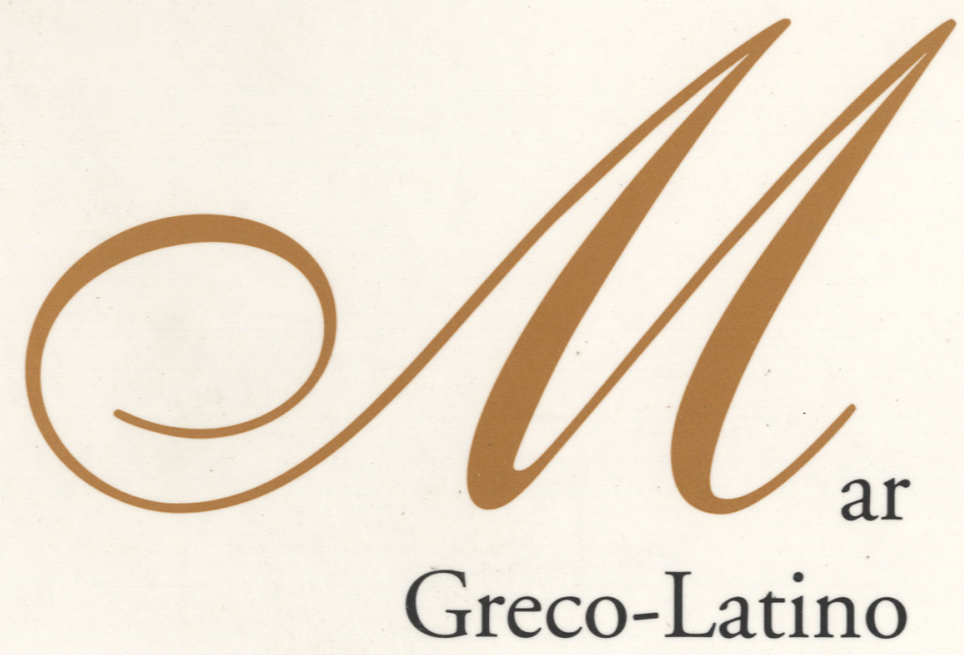

- colmbra 2006 


\title{
BUSCAR O MAR PELA MONTANHA
}

\author{
Antonio Sartori
}

Università degli Studi di Milano

À LA MER PAR LES MONTAGNES - La mer et les montagnes ne furent pas des lieux incommunicables dans les temps anciens, si non dans la littérature: réciproquement inconnus, comme dans le mythe de l'Odysseus d'Homère, qui aurait dû atteindre à des lieux si éloignés de la mer que personne n'y aurait connu les symboles de la mer; ou idéalement opposés, comme la montagne (du Purgatoire) qui repoussa les efforts de l'Ulysse de Dante Alighieri. Mais, dans la Méditerranée gréco-romaine au moins, la mer et la montagne furent le théâtre de plusieurs échanges. Le fond Ouest de la plaine du Pô eut plus des contacts (militaires, commerciaux, culturels et religieux) avec la mer de Marseille par les Alpes, que, le long des fleuves, vers la mer Adriatique. Et, du haut de la montagne, par exemple par le Trophée de La Turbie - grand monument et impérieuse inscription ensemble - la voix de l'empereur pouvait résonner ou être vue et reconnue concrètement jusqu'à l'horizon de sa mer, mais aussi idéalement jusqu'aux extrêmes limites du monde.

Per la comune tranquillità dei pazienti ascoltatori, con il titolo del moi contributo incomincio e finisco il mio exploit in lingua portoghese, e me ne scuso vivamente. E tuttavia mi è sembrato significativo principiare con un 
adattamento del celeberrimo motto di Cristóvão Colombo "buscar Oriente per Occidente" oppure "buscar Oriente e tomar Occidente", perché questo vorrebbe essere l'atteggiamento conduttore del mio breve intervento.

Prima, però, un ringraziamento particolare anche da parte mia vada agli organizzatori di questo incontro internazionale, in cui, in coppia con l'amico e collega professor Alfredo Valvo, rappresentiamo forse è troppo, ma segnaliamo la presenza della componente italiana nella nostra comune attività di ricerca: un ringraziamento doveroso, ma anche calorosamente spontaneo.

E da buon italiano, da dove meglio incominciare che da Dante Alighieri e dal suo Ulisse? - un Ulisse poco Ulisside ma molto dantesco, a dire il vero.

Sì, perché nella Comedia Ulisse $^{(1)}$ non giunge mai a trovare pace nella sua Itaca: dopo l'ultimo impedimento in cui si era incagliato, sottrattosi ormai alle panie di Circe, egli volge altrove le sue vele volontariamente, per un fuoco interiore che lo spinge perennemente a "seguir virtute e canoscenza".

Ulisse il navigatore continua non più le sue peregrinazioni fatali, cui era stato costretto, ma sponte sua un suo viaggio interminabile, il viaggio di una vita in cui mettersi alla prova fino all'estremo: ancora una volta sul mare, sulle superficie d'acqua che di tutti gli ambienti ecologici sono le più connaturate con l'ignoto, vaste e interminate quant'altre mai: sul mare, ma oltre - ed è l'oltre che qui più conta - oltre le barriere volute dal dio, quelle che concretamente e umanamente definiscono gli spazi umani; oltre, e dunque verso una meta ormai indeterminata, che poi si materializzerà in forma tragicamente conclusiva.

Con quel tanto di colombiano appunto, ma ante litteram, che traspare già dall'affrontare l'ignoto in termini aleatori, ma che si sostanzia più concretamente nella direzione presa e mantenuta poi per mesi, verso il sudest,

\footnotetext{
(1) Inferno, canto XXVI, 90-142. Inutile peraltro ricordare che Dante non aveva cognizione diretta di Omero.
} 
bordeggiando le coste atlantiche dell'Africa. La cui conclusione infine, quasi più voluta e ricercata che fatale, si discosta nettamente e tuttavia tanto dal futuro consapevole realismo di Colombo, quanto dalla mitica profezia vagheggiata per l'Odysseus di Omero.

Ed ecco qui una contrapposizione - una contrapposizione, e dunque nulla di armonicamente integrato - molto netta fra mare e montagna: ma quale mare e quale montagna!

Questo mare è di per sé infinito, fuori dai limiti concreti imposti dagli dei alle esperienze umane; e la montagna che Ulisse intravede di lontano non è una comune montagna, fisico e pedologico corrugamento levato sulla faccia della terra, ma trascende allegoricamente a situazione ideologica, a stato d'animo: è la montagna del Purgatorio, una nova terra da cui infine un turbo nacque ... infin che il mar fu sopra noi richiuso ${ }^{(2)}$. Fine definitiva e fatale, di incomunicabilità coatta tra mare e montagna, di due mondi fra i quali la spinta alla conoscenza è stata esasperata fino all'estremo ed oltre, fino all'annientamento consapevole e fatale nello stesso tempo.

Epperò qui dunque mare e montagna, sublimati da concrete realtà ecologiche qual erano a ideali e miti, sono in contrasto per così dire insanabile, contrapposti ed inconciliabili, intangibile l'una se affrontata attraverso l'altro.

All'opposto, si può ben dire, l'Odysseus Omerico: il gran viaggiatore, ma obtorto collo, prima nella speranza sempre delusa e procrastinata per il volere divino di raggiungere infine la sua patria; poi, altrettanto obtorto collo, costretto ineluttabilmente dal suo fato a proseguire ancora nel suo peregrinare lungo percorsi, quelli acquatici, neppure segnati, verso una pace forse definitiva: ma non sappiamo neppure quanto sua pace personale ed intima -

(2) Inf., XXVI, vv. 137-142: “... ché de la nova terra un turbo nacque, | e percosse del legno il primo canto. | Tre volte il fe' girar con tutte l'acque: | alla quarta levar la poppa in suso | e la prora ire in giù, com'altrui piacque, | infin che 'l mar fu sopra noi richiuso". 
e quale pace poi ${ }^{(3)}$, ma almeno pace infine nei confronti degli dei, da sempre adirati con lui e ancora non paghi, giusta la profezia di $\operatorname{Tiresia}^{(4)}$.

Questa infatti la profezia risolutiva: volgere le spalle al mare, raggiungere per strade polverose una terra tanto lontana o appartata dal mare che neppure se ne sarebbero riconosciuti i suoi simboli, tanto da scambiare un remo (gr. eretmón) per un ventilabro (athereloigós), che così suona anche in portuguese, là dove nessuno vi avrebbe mai visto una nave, di cui i remi sono le ali; e là dove neppure si sapessero insaporire le vivande con il sale. Solo allora, celebrati i debiti sacrifici a Poseidon, Odysseus sarebbe potuto tornare a casa, davvero finalmente questa volta, a placarsi, anzi ad essere mollemente "consunto al fin da una lenta vecchiezza, finché morte sopravverrà placida e dolce, E beate vivran le genti intorno": un buen retiro insomma, da pensarsi comunque improbabile e forse persino sgradito per un sempre dinamico Odysseus, neanche fosse ormai un pensionato, un "reformado".

\section{Al mare per la montagna}

Ed ecco dunque il problema legato al tema. Concretamente, storicamente, sarebbe stata mai possibile una terra tanto dislocata, tanto separata, tanto anomala rispetto alle esperienze, tutte e tante, le poliedriche esperienze di Ulisse uomo di mare?

In assoluto non si potrebbe escludere - forse allora più che oggi l'esistenza di qualche plaga tanto remota sulla faccia del pianeta, dove la

\footnotetext{
(3) Ma quale pace sarebbe dovuta essere, per un navigatore come lui, e se mai l'avesse raggiunta, così come non sappiamo perché Omero non lo dice? Forse il contrappasso punitivo d'essere messo alla prova ancora - e quanto? - in un ambiente nuovo, a lui del tutto estraneo, solida ma polverosa terra per un frequentatore degli aperti spazi acquorei? Oppure l'acquietamento infine nel cessare di un'eterna angoscia, non più scosso dai flutti vendicativi, ma snervato da una lunga fatica itineraria in un mondo del tutto statico? Per non dire più banalmente della consolazione - e quanto soddisfatta? - del cambiare radicalmente vita?

(4) Odyss. XI, vv. 126-134, poi ripresi in XXIII, vv. 265-287. Cfr. M. Zambarbieri, L'Odissea com'è. Lettura critica. I, Milano 2002, pp. 827 ss.
} 
montagna, lontana per misure itinerarie come per altitudine, si possa o si potesse ben ergere in contrapposizione anche concettuale con il mare.

Ma nella realtà del mondo classico, quella a cui in questi giorni ci dedichiamo, no certamente, tanta lontananza non sarebbe mai potuta essere possibile.

E tanto più nelle aree di cui ho più diretta esperienza di studio, l'Italia del Nord, la regione alpina e, per estensione, l'odierna Europa centrale.

In fondo, le distanze itinerarie europee non hanno mai raggiunto entità tali da rendere del tutto impraticabile ogni connessione con le coste; e infatti ben presto anche proprio le aree più impervie, dislocate dal mare più che per distanza per altitudine, e dunque mai marginali ma centrali per quanto estranee od evitate, furono, perché dovettero essere, percorse da correnti di collegamento che le unirono in un network efficiente ed efficace: e non si dice dei non più mitici percorsi protostorici delle vie dell'ambra, per esempio, da mare a mare, dal mar Baltico all'Adriatico, ma dei più funzionali tracciati stradali storici, che di tronco in tronco connesso irretirono tutto il continente.

Il che tuttavia non significa che ogni area, solo perché percorsa da vie interregionali, dovesse di fatto essere coinvolta nella sua totalità in un fenomeno di globalizzazione ante litteram. Perché ci fu pure chi - il fenomeno è antropico e forse sociale, non ecologico - non la volle riconoscere anche nell'antichità: prima quanti si opposero(5) alla violazione del proprio territorio nel vedervi attrezzare vie che li oltrepassavano verso mire lontane (quasi un sentore precoce di certe anacronistiche velleità di oggi di opposizione contro gli assi ferroviari europei della TAV?); poi quanti disturbarono i transiti o ne pretesero ricadute positive forse non del tutto illegittime, con la prepotente imposizione di pedaggi o con il coinvolgimento

(5) Nello specifico dell'area, G. Brizzi, "Scene di guerra in montagna", in Gli antichi e la montagna. Les anciens et la montagne, Atti del Convegno. Aosta 1999" (a c. di S. Giorcelli Bersani), Torino 2001, pp. 199-211 
più collaborativo e redditizio di guide o di fornitori di servizi locali( ${ }^{(6)}$; infine, o tutt'insieme, quanti se ne estraniarono, cercando e difendendo un aureo isolamento.

Da cui tuttavia infine vennero tratti - o anche attratti - dagli interventi mirati di quanti, entità politiche ormai sovraregionali, di quelle vie e della loro migliore agibilità avevano bisogno.

Per scoprire le mie carte, a titolo di esempio, mi riferisco ovviamente ad uno degli episodi, ma prolungato ${ }^{(7)}$, degli attriti fra le aree alpine con i loro abitanti e la "grande potenza", Roma, in via di farsi dominante unica: la soluzione conclusiva del problema dei Salassi nell'odierna Val d'Aosta ${ }^{(8)}$.

Non è un caso che qui la "soluzione finale" del problema dei cosiddetti "popoli alpini", un problema non di poca durata e neppure di importanza minore, fu escogitata con qualcosa di "marino" in certo senso.

Dopo una fastidiosa, perché reiterata e violenta, opposizione guerrigliera ai transiti, dopo l'estorsione e la richiesta intimidatoria di pedaggi, cui dovettero sottostare interi corpi d'armata (da Decimo Bruto alle truppe di Augusto stesso) per buona parte dell'ultimo secolo della repubblica, la mossa vincente dei Romani fu l'embargo dell'importazione di sale.

Omero aveva potuto immaginare, come estrema diversità culturale, la non conoscenza del sale e dei suoi usi, dovuta alle grandi distanze dal mare, in realtà si sa e qui si vede che nessuna comunità avrebbe mai potuto farne a meno.

\footnotetext{
(๑) A. Sartori, "I privilegi dell'altura", in Gli antichi e la montagna, cit., pp. 107 ss.

(7) G. Oberziner, Le guerre di Augusto contro i popoli Alpini, Roma 1900

${ }^{(8)}$ Può essere singolare - per quanto debbano contare analogie diacroniche troppo anacronistiche - che forme di rivendicazioni di una specificità territoriale e antropica non rimasero confinate alle esperienze dei Salassi, ma debordarono ampiamente fin quasi all'oggi con singolari affermazioni: cfr. C. Bocca, M. Centini, Sulle tracce dei Salassi: origine, storia e genocidio [! ] di una cultura alpina, Ivrea 1995, pp. 63 ss. Traccia concreta e tuttora visibile ne rimane nella stele commemorativa rizzzata davanti alla Porta di Augusto all'ingresso di Aosta, già Augusta Praetoria, che così recita: Le Salasse longtemps | defendit ses foyers. | Il succomba. | Rome victorieuse | ici | deposa ses lauriers. | Cette plaque commemorative | disparue en 1914 a été replacée | en 1985 par le Comité des | Traditions Valdôtaines.
} 
Tanto più le popolazioni alpine, che erano geograficamente le più estranee dal mare, ma che avevano fondamentale base economica nella zootecnia, con un evoluto sfruttamento a rotazione dei pascoli ad ogni quota $^{(9)}$; per le quali in questa storica occasione congiunturale, l'embargo del sale fu esiziale, perché troncò la essenziale produzione casearia, sostentamento di base ed unica concreta moneta di scambio con l'economia delle pianure.

Del sale, la cui sola fonte di approvvigionamento, almeno in una vasta area europea circostante, era costituita dalle saline marine costiere, per quanto distanti, quelle ad evaporazione naturale in bacino: del tutto prevalente per area e per quantità, rispetto ad una sporadica produzione per riduzione termica su fuoco vivo, laboriosa e dispendiosa e molto locale, e rispetto pure all'estrazione di sale ipogeo - quello che si dice salgemma - che era più presente nelle regioni più nordorientali ${ }^{(10)}$.

Il sale marino: che non proveniva tuttavia dalle bassure della pianura padana, spalancata verso il mare Adriatico ma troppo di lontano, ma che giungeva dalle saline marsigliesi, di quella colonia greca in terra gallica che, benché transalpina, ebbe l'occasione, o il compito, o il merito di avvicinare una parte importante dei popoli alpini al mondo evoluto.

Senza interpretare fantasiosamente un'Alpis Graia - l'odierno col du Petit Saint Bernard - come indizio di improbabili transiti organizzati di Greci, per non dire di mitiche escursioni di $\operatorname{Ercole}^{(11)}$, perché più prosaicamente Alpis

\footnotetext{
(9) A. Niederer, "Economia e forme tradizionali di vita nelle Alpi", in Histoire et Civilisations des alpes, II. Destin humain, Lausanne $1980=$ tr. it. Storia e civiltà delle Alpi. Destino umano (a c. di P. Guichonnet), Milano 1984, pp. 9-104.

(10) L. Pauli, Die Alpen in Frübzeit und Mittelalter, München 1980 (trad. it. Le Alpi: archeologia e cultura del territori. Dall'Antichità al Medioevo, Bologna 1983), pp. 272 s.; M. Besnier, in DA IV, s.v. sal; Ch. Singer et alii, A History of technology, I, Oxford 1954 (trad. it. Storia della tecnologia, I, Torino 1966) pp.259 ss.; A. Riparbelli, "Sorgenti salate e miniere di sale", in Les eaux thermales et les cultes des eaux en Gaule et dans les provinces voisines. Actes du Colloque, septembre 1990, Tours-Turin 1992, pp. 349-377.

(11) R. Dion, "La voi Heracléenne et l'itinéraire transalpin d'Hannibal", in Hommages à Albert Grenier, Bruxelles 1962, pp. 527-543.
} 
Graia deriva da una componente lessicale celtica legata alle montagne innevate - è un dato di fatto ben acquisito che la produzione numismatica “celtica"(12) (un'entità prestatuale, quella dei Celti padani, che tuttavia ebbe strutture organizzative elaborate quanto oggi ancora indistinte $\left.{ }^{(13)}\right)$ fu radicalmente e progressivamente mutuata ${ }^{(14)}$ dagli influssi - certamente culturali e funzionali, ma pure economici - degli insediamenti greci di Massalía/Massilia e dell'area circostante ${ }^{(15)}$.

Di cui si può riconoscere una spontanea e lunga sopravvivenza fino all'oggi, nel realizzarsi prima della plurisecolare cultura occitana, tipicamente incentrata sul doppio versante alpino(16), e, nel più recente passato, nella persistenza di pur modesti scambi commerciali con l'oltralpe (ad esempio, ricordo che il tradizionale commercio, un tempo ambulante, del pesce conservato nell'odierno Piemonte non giunge dalle pianure, ma... scende dalle valli(17), e che l'arancia e anche una mela locale ${ }^{(18)}$ sono tuttora dette "purtigàl", come provenienti dall'occidente, non dal sud).

Ed ecco dunque un primo modo di guardare verso il mare, di avere contatti con il tramite acquatico verso il mondo.

Ma attraverso le montagne, qui fra l'altro di modesto sviluppo in larghezza, e tuttavia impervie $\mathrm{e}^{(19)}$; e in un indirizzo all'apparenza innaturale, perché nella penisola italica le pianure del Nord hanno una spontanea e larghissima bocca aperta al mare nella direzione opposta, verso oriente: ma molto, troppo,

\footnotetext{
(12) A. Pautasso, Le monete preromane dell'Italia settentrionale, Varese 1966, pp. 33 ss.

(13) V. Kruta, "I Celti", in Italia omnium terrarum alumna, Milano 1988, pp. 287 ss.

(14) E.A. Arslan, "I Transpadani", in I Celti, Milano 1991, pp. 461-470.

(15) Marseille grecque et la Gaule. Actes du Colloque international d'histoire et d'archéologie et du 5. Congrès archéologique de Gaule meridionale. Marseille, 18-23 novembre 1990 (eds. M. Bats et alii) Aix-en-Provence 1992.

(16) Le rayonnement de la civilisation occitane à l'aube d'un nouveau millénaire. Actes du be Congrès international de l'Association internationale d'études occitanes, 12-19 septembre 1999 (eds. Georg Kremnitz et alii), Wien 2001.

(17) D. Crestani, Anciùe e caviè 'd la Val Mairo. Mestieri del'emigrazione stagionale alpina, Cuneo 1992.

(18) I dati tipologici in Atlante pomologico biellese (www.pronaturabiellese.org/ ScPomo.html).

(19) W. Woodburn Hyde, Roman Alpine Routes, Philadelphia 1935.
} 
dislocata rispetto a queste aree e aperta sì, ma verso un mare interno come l'Adriatico.

Ora è ben vero che il gran fiume Po - grande per il contesto italico e forse anche in virtù delle sensibili diversità climatiche ed ecologiche di allora, ma sempre non gran cosa rispetto ai corsi d'acqua centroeuropei - è ben vero che il Po percorreva in tutta la sua lunghezza la vasta pianura ${ }^{(20)}$.

Ma le fonti antiche ci dicono che le sue acque mai troppo violente le si risalivano navigando per un lungo tratto, non che le si discendessero: perché il fiume era tramite e via di penetrazione dal mondo, per non dire dal mondo "civile", verso un interno volta a volta da colonizzare, o da coinvolgere economicamente, o da godere.

E penso alle colonie di Placentia e di Cremona, le prime di fronte al pericolo gallico, isolate all'apparenza entro un mare di genti ostili, invece al mare collegate e dal mare sostenute proprio per via d'acqua sul $\mathrm{Po}^{(21)}$, prima di essere allacciate con la via Emilia.

E penso all'affermazione di Plinio $^{(22)}$ che imbarcazioni di rilevanza commerciale ed economica sapevano risalire il fiume fino alle porte di Augusta Taurinorum o almeno all'antica Bodincomagus e poi Industria: risalire appunto, ancora una volta, per un import mirato ai consumi interni, meno per un export da quelle aree, modesto e rivolto verso altre direzioni.

Così come aveva risalito il Po, ma solo per breve tratto, ed il Mincio suo affluente, il phaselus ille, la snella imbarcazione orientale - da diporto diremmo oggi - che Catullo(23) aveva trasferito nel suo buen retiro sul lago di

\footnotetext{
(20) R. Chevallier, La romanisation de la Celtique du Pô. Rome 1983, pp. 22 ss.; G. Uggeri, "La navigazione interna della Cisalpina in età romana", in "AAAd" XXIX (1987), pp. 305-354; G. Uggeri, "Le vie d'acqua nella Cisalpina romana", in Optima via. Atti del Convegno Internazionale 'Postumia' Cremona 13-15 giugno 1996 (a c. di G. Sena Chiesa, E.A. Arslan), Milano 1998, pp. 73-84

(21) Strab.V, 1, 11, ma forse solo come terminal di un tratto del cursus publicus, e comunque ben posteriore a questi tempi, che non come limite di navigabilità.

(22) Plin. n.h., III, 123.

${ }^{(23)}$ Catull c. 4, 1.10.15
} 
Garda: risalito, ma di poco, per giungere e lì fermarsi definitivamente, solo in vista delle montagne, ma ancora ben lontano.

\section{Dal mare alla montagna}

E che di quel mare, l'Adriatico, ancora si sentisse l'eco in tutta l'area veneta - pianeggiante per lo più, le montagne innalzandosi sui margini - lo dimostra la presenza sparsa di testimonianze - epigrafiche per lo più, le più concrete del culto a Neptunus.

Dico in breve, perché non è questo il tema d'oggi: ma la presenza di Neptunus ha qui un suo significato ${ }^{(24)}$ : la sua concentrazione verso oriente, intorno alla gran bocca della pianura sull'Adriatico, lo dichiara per quel che è, dio acquatico, ma di mare; per quanto lo si voglia oggi riconoscere come divinità delle acque, di tutte le acque, e poi, con estensione forse troppo facile, delle acque salutari.

Il che non mi sembra proprio nel Norditalia: troppo scarse le testimonianze epigrafiche appunto, e non molto aggiungono le insignificanti presenze di bronzetti( ${ }^{(25)}$ di Neptunus, neppure troppo riconoscibili, e che per dimensioni esigue, per facile riproducibilità, per trasporto occasionale, possono essere solo labili indizi di culti davvero locali. Scarse testimonianze quelle epigrafiche, che si riducono ancora, se si considera che anche le due più consistenti da Como, e dunque ben lontane dal mare, tornano ad essere per qualità mimetica o per nostalgia - rievocazioni di culti marittimi: l'una ${ }^{(26)}$, un altare pro salute et incolumitate, che si rifà certo alla navigazione sul lago

\footnotetext{
(24) A. Arnaldi, Ricerche storico-epigrafiche sul culto di 'Neptunus' nell'ttalia romana, Roma 1997.

${ }^{(25)}$ M. Bolla, "Bronzetti romani di divinità in Italia Settentrionale. Alcune osservazioni", in Bronzi di età romana in Cisalpina: novità e riletture, Atti della 32. settimana di studi aquileiesi 2001, in "AAAd" LI (2002), pp. 119 s.

${ }^{\left({ }^{26}\right)}$ Arnaldi, Ricerche, cit., pp. 180 ss. num. 37 (CIL V, $5258=$ ILS, 3283): Neptuno et $\mid$ dis aquatilibus | pro salute et | incolumit(ate) v. s. l. m. | C(aius) Quart(ius) Secundin(us). Cfr. A. Sartori, Parlano anche i sassi. Per un'intepretazione dell'Epigrafia latina, Milano 2001, p. 58.
} 
di Como che sa essere pericoloso del suo; l'altra una gran lastra celebrative ${ }^{(27)}$ che solennizza certi provvedimenti locali per le feste Neptunalia, quelle più tradizionali: il che ben si adatta con la presenza o il radicamento, forse oggi più sicuro che un tempo, di quei cinquecento greci che Cesare trapiantò a Como proprio con funzioni naviganti.

Testimonianze, dunque, tutte legate all' "altro" mare, all'Adriatico e per i tramiti più spontanei, la gran pianura e il suo sistema idrico. Il che però non vale per l'altare ${ }^{(28)}$ dei piscatores di Pedo: alle soglie delle valli alpine più riposte (forse non a caso sede di una statio della Quadragesima Galliarum, come a dire proiettata funzionalmente verso l'esterno, ma dell'oltralpe) e certamente disconnessa da ogni rete di collegamenti fluviali, perché troppo a monte.

La ricca sequela di sette o sei offerenti (secondo certe varianti inerpretative), ma tutti con nomi in certa misura indigeni, li connette strettamente con la realtà locale; l'articolata raffigurazione del dio, in piedi sulla barca con tridente e corno, torna a farne risentire odori di mare, che però qui è quello più vicino e qui più esemplare, quello del marsigliese golfo del Leone.

Per non dire poi di un particolare, forse modesto, che mi ha sempre incuriosito: la presenza di elementi marini nella simbologia, per esempio in quella funeraria: quanto i volonterosi lapicidi della mia Transpadana ebbero cognizione concreta e visiva di quei delfini che realizzarono con fantasia

(27) CIL V, 5279 = ILS 6728: ... ex quorum [scil. (sestertium nummum) (quadragies) (milium)] reditu quotannis per Neptunalia oleum in campo et in thermis et in balineis omnibus quae sunt Comi populo praeberetur... Cfr. A. Sartori, "Quadro dell'epigrafia comasca", in Novum Comum 2050. Atti del convegno, Como 1993, pp. 253 s.; A. Sartori, Le iscrizioni romane. Guida all'esposizione (Musei Civici di Como), Como 1994, p. 37 sch. Po10 (num. inv. L151); A. Sartori, “... per Neptunalia oleum in campo", in Pro poplo Arimenese (a c. di A. Calbi e G. Susini), Faenza 1995, pp. 564 ss.

(28) Arnaldi, Ricerche, cit., pp. 189 ss. num. 40 (CIL V, 7850 = ILS, 3287); E. Culasso Gastaldi, G. Mennella, Pedona, in Suppl. It., n.s. 13, Roma 1996, p. 310: ((Neptunus stans in cymbula, tenens cornu et fuscina)) Neptuno sac(rum) | Maximus Teurius | vicarius Metelae | Danius Carb[ - - ] | Vibius Velagenius Pesa | Parra Enicius | Miranius Carb [- - ] | Silvanus Velagenius Ebelin [ - - ] | Lasser Metela Edanius Car[ - - ] | Maximus Minatius Carb[ - - ] | Secundus Enicius Parrae f(ilius) Barg[---]| piscatores l(ibentes) m(erito). 
estrosa ed approssimata? Mai uno ne videro al naturale, eppure l'opinione pubblica li gradiva, la clientela li pretendeva

\section{La montagna sul mare}

Infine, il mare come cassa di risonanza, come luogo di esposizione privilegiata.

La conclusione dell'operazione Salassi rientrava nella soluzione finale del complesso e generale problema dei "popoli alpini", fin qui, quale più quale meno, tutti riottosi o ben poco collaborativi, e della sicurezza dei transiti attraverso i territori da loro abitati, ormai resa essenziale dalla trasformazione della politica romana da peninsulare a transalpina o, per dirla un po' anacronisticamente, europea.

Non per nulla nel monumentum Ancyranum, testamento politico ed ideologico dell'Augusto, l'operazione viene sintetizzata non prima ma dopo le più prestigiose iniziative nelle province: un'operazione di razionalità e di polizia, che sanava ripetute occasioni di disturbo: Alpes a regione ea, quae proxima est Hadriano mari ad Tuscum pacari feci. ${ }^{(29)}$ Cui seguì - ed è questo il documento a cui penso - l'erezione del cosiddetto Tropeum Alpium, con la ripresa, in forma monumentale, delle stesse espressioni analiticamente comprendenti le 44 gentes alpinae coinvolte, così come recita la grande iscrizione ${ }^{(30)}$ : quod eius ductu auspiciisque gentes Alpinae omnes quae a mari Supero ad Inferum pertinebant sub imperium populi Romani sunt redactae.

In un recente convegno - Epigrafia di confine, confine dell'epigrafia, Bertinoro 2003 - ci siamo ritrovati a nostra insaputa, Jean-Michel Roddaz ed

\footnotetext{
(29) $R . g ., 26,3$.

(30) CIL V, 7817; cfr. Plin. n. b. 3, 136. M.J. Formigé, Le trophée des Alpes (II Suppl. 'Gallia'), Paris 1949 ; Id., "La dédicace du Trophée des Alpes (la Turbie)", in "Gallia ” 123 (1959), pp. 101 ss. J. Šašel, "Zur Erklärung der Inschrift am Tropaeum Alpium (Pline NH, III, 136-137; CIL V, 7817)", in "Živa Antiquité Vivante" 22 (1972), pp. 135-144.
} 
io ${ }^{(31)}$, in singolare coincidenza di interpretazione delle funzioni dei Tropaea di età augustea. Monumentali strutture edilizie - e la monumentalità ne era carattere fondamentale - distribuite sui confini non in quanto limiti giuridici, ma come indici estremi di espansione, votati a raggiungere le ultime propaggini dell'ecumene. Essi avevano complesse funzioni di autoritaria presenza politica e militare con la loro mole, di definizione conclusiva dello spazio umano con la loro collocazione, di comunicazione magistrale e impositiva "verso il nulla", come ebbi a dire, volta intenzionalmente verso là, teoricamente la fine del mondo, dove nessuno avrebbe potuto averne cognizione, o dove misurarsi invece con gli spazi interminati di ben più che l'ecumene.

E il Tropaeum Alpium posto a La Turbie, come connetterlo con tutto ciò? Esso non è certo ai confini del mondo, ma affacciato - affacciato appunto su un tratto di mare aperto fra i più frequentati.

Una posizione simbolicamente inserita in un quadrante universale: la sua vasta iscrizione comprendeva gentes Alpinae anche delle Alpi più orientali, all'estremità opposta dell'Italia, ma esso era collocato sulla via Iulia Augusta che puntava verso l'occidente e si collegava idealmente con i tropea dei Pirenei e con quelli atlantici; ancora, la sua vasta iscrizione elencava decine di gentes Alpinae, ma esso si stagliava sull'alto della montagna, rivolto però non verso il nulla ma verso l'indeterminatezza degli spazi marini: tutt'altro che deserti, tuttavia, solcati anzi da un reticolo di rotte di essenziale importanza.

Non è mancato chi, esaltandone la posizione non indifferente come anche la grande mole, vi ha riconosciuto una funzione persino tecnica di faro o di punto di riferimento per la sicurezza delle rotte; un traguardo, dunque, cui

\footnotetext{
(31) "Epigrafia di confine, confine dell'epigrafia. Atti del Colloquio AIEGL-Borghesi 2003 (a c. di M.G. Angeli Bertinelli, A.Donati)", Faenza 2004: A. Sartori, "Titulus clamans in deserto: l'epigrafia verso il nulla", pp. 23-32 (spec. pp. 28 s.) e J.-M. Roddaz, "Tropea in finibus: l'épigraphie et l'exaltation de la conquête aux confins de l'Empire", pp. 33-47 (spec. pp. 41 s.).
} 
tutti erano indotti a guardare con attenzione e che pertanto si imponeva su larghi spazi, fino all'orizzonte ed oltre, con la sua presenza fisica, ma anche con il messaggio - o il significato simbolico - che esso veicolava e che da qui aveva modo di essere divulgato sulle grandi distanze anche senza di necessità essere compitato o letto e compreso da vicino.

Essenza magistrale, una volta di più, della comunicazione epigrafica, in grado di mettere in relazione gli estremi più diversi, persino gli illetterati e gli analfabeti da un lato ${ }^{(32)}$, imponendosi con la sua presenza, o come, in questo rapporto di osmosi fra mare e montagna, persino chi neppure vedesse l'iscrizione, ma pur di lontano riconoscesse la sagoma e il profilo del monumento e rievocasse dalla sua esperienza ma idealmente quanto già noto per altre vie.

Una funzione persino ecologica, dunque, quella dell'epigrafia, nel connettere ambienti tanto diversi o fin contrapposti come mare e montagna: un merito in più dei suoi tanti, che pure oggi debbono misurarsi con la sconsiderazione comune, ahimè sempre più diffusa immeritamente ${ }^{(33)}$.

\footnotetext{
(32) A. Sartori, "L'adlocutio adrianea sulla pietra a Lambaesis. Come e perché", in "Loghios anèr. Studi di antichità in memoria di Mario Attilio Levi (a c. di P.G. Michelotto) ", Milano 2002, pp. 351-365; M. CORBIER, "L'écriture dans l'espace public romain", in "L'Urbs. Espace urbain et bistoire. Ier s. av. J.C.- IIIe s. aprJ.C.", Rome 1987, pp. 57 ss.; G. SusinI, "Compitare per via. Antropologia del lettore antico: meglio, del lettore romano", in "Alma mater studiorum", I,1, pp. 105 ss.; Id., "Le scritture esposte", in "Lo spazio letterario di Roma antica. 2", Roma 1989, pp. 271-305; M. CORBIER, "L'écriture en quête de lecteurs", in "Literacy in the roman World (eds. M. Beard et alii)", Ann Arbor (Mi), pp. 99 ss.; A. Sartori, "Le forme della comunicazione epigrafica", in "Monumenti sepolcrali romani in Aquileia e nella Cisalpina. XXVI Settimana di Studi Aquileiesi 1995" =AAAd, XIIII, Trieste, pp. 39-65; Id., "Presenza e funzioni delle epigrafi esposte nella città romana", in "Ciudades privilegiadas en el Occidente Romano. Actas del Congreso Intern. Sevilla 1996 (ed. J. Gonzalez)", Sevilla 1999, pp. 117-126; Id., "Tituli da raccontare", in "Scripta volant? Atti del $2^{\circ}$ incontro di Dipartimento sull'epigrafia. 5 maggio 2004 (a c. di A. Sartori)", Milano 2005, pp. 93 ss.; Id., "Le iscrizioni latine litterature de rue", in "Urbs aeterna. Actas y colaboraciones del Coloquio Internacional 'Roma entre la literatura y la historia' (Hom. a la profesora C. Castillo). Pamplona 2003 (eds. C. Alonso del Real et alii)", Pamplona 2003, pp.742 ss.

(33) Il presente studio si inserisce in un programma interuniversitario PRIN, cofinanziato dal MIUR, di cui lo scrivente è responsabile locale e la prof. Angela Donati (Università degli Studi di Bologna) è coordinatore nazionale.
} 